Research Article

\title{
Multimotor Improved Relative Coupling Cooperative Control Based on Sliding-Mode Controller
}

\author{
Changfan Zhang $\left(\mathbb{D}\right.$, Mingjie Xiao $\left(\mathbb{D}\right.$, and Jing He ${ }^{1}$ \\ College of Electrical and Information Engineering, Hunan University of Technology, Zhuzhou 412007, China \\ Correspondence should be addressed to Jing He; hejing@263.net
}

Received 23 March 2020; Accepted 2 May 2020; Published 20 May 2020

Guest Editor: Xiao Ling Wang

Copyright () 2020 Changfan Zhang et al. This is an open access article distributed under the Creative Commons Attribution License, which permits unrestricted use, distribution, and reproduction in any medium, provided the original work is properly cited.

\begin{abstract}
To provide cooperative control under complex working conditions of a filling multimotor system, this paper proposes a relative coupling control strategy with a switching system structure. Firstly, a multistation transmission system composed of a filling motor and a transfer motor is designed according to different filling processes. Secondly, a stable sliding-mode surface common to the multimotor system is selected, and an equivalent sliding-mode controller corresponding to each motor is designed. Thirdly, public Lyapunov stability theory is used to prove that the switched system can move from any initial state to the common sliding surface of the system, thereby ensuring the asymptotic stability of the entire system. Simulation results show that this method has a more significant control effect on the system error of each motor in comparison with the traditional relative coupling control structure.
\end{abstract}

\section{Introduction}

As a major technical problem in filling production, multimachine collaborative control is widely explored by researchers in the food packaging industry $[1,2]$. Multimotor synchronous control is an important part of multimachine collaborative control technology for filling production. A long period of research produced numerous results $[3,4]$. However, two main problems remain in the filling process of thick sauces and sticky foods. First, the system starts and stops frequently during the filling operation. Second, when the filling operation is completed, the filling motor exhibits an abrupt load torque. In view of the two problems, this study focuses on how to realize the multimotor synchronous control of the filling system under complex working conditions.

In terms of synchronous topology, parallel synchronous control and master-slave synchronous control are noncoupling structures [5]. The motors lack connection, and the synchronization performance is easily affected by external disturbances and load torque changes. Thence, Zhang et al. [6] proposed a virtual total axis control structure based on an equivalent load observer, therefore addressing the difficult synchronization between the multiaxis system of the printing press when the load disturbance is large. However, they did not consider the coordination between multiple variables. For high-speed EMU, Yang et al. [7] established a multiadaptive neural fuzzy inference system (ANFIS) model and designed a predictive controller to achieve the EMU operational control. Zhao and Zhang [8] first proposed the relative coupling control structure, but adding a speed feedback module would complicate its structure as the number of motors increased. Thus, to solve these problems, Shi et al. [9] improved the relative coupling control strategy by introducing an additional speed controller and simplified the system structure. Sun et al. [10] adopted a fuzzy SMC based on ring-coupling synchronous control structure to enhance the reliability and control accuracy of the multimotor drive system. Zhang et al. [11] proposed a switching structure control method based on state switching and designed a nonlinear SMC to achieve synchronous control of the printing press multiaxis system in the case of time delay. $\mathrm{Li}$ et al. [12] proposed a second-order adaptive SMC method based on average relative coupling. The adaptive law 
overcame the effects of bounded external disturbances and parameter perturbations on the system, thus improving robustness. Huang et al. [13] proposed an external coercion mechanism to analyze the problem of extensive cooperation. By modeling the agent on a simple conventional network, it proved that the mechanism has a significant promotion effect on cooperation.

In terms of synchronous control algorithms, researchers are prone to enhancing control accuracy by improving the algorithm [14]. Li et al. [15] introduced the adaptive slidingmode algorithm to the ring-coupling control strategy and solved the poor synchronization performance of multiple motors in the case of uncertain perturbation of system parameters. Xia et al. [16] used a two-degree-of-freedom PI velocity controller and improved the effect of noise and time delay on synchronization performance by using Kalman filtering. He et al. [17] used a sliding-mode controller to achieve multiaxis servo cooperative control under variable proportional conditions. Yang et al. [18] studied the eventtriggered consensus (ETC) problem based on multiagent systems with linear dynamics and implemented multiagent consistency control by using the ETC controller. Zhang et al. [19] solved the problem of distributed robust cooperative control of multimotor systems with unknown compound disturbances, and they further proposed a distributed robust cooperative control algorithm based on a disturbance observer. Xia et al. [20] combined a cross-coupling structure with a sliding-mode control algorithm and used an exponential approach law and a saturation function to propose a synchronous control strategy. Wang et al. [21] proposed an adaptive and robust $H^{\infty}$ control strategy using the combination of a robust tracking controller and a distributed synchronous controller which implemented load tracking and synchronous control of a multiservo system. Liu et al. [22] added a mismatched relay node to the complex motor network to reduce the coupling threshold for synchronization of the entire complex motor network and thus improve the synchronization performance of the entire network. Acknowledging that the status information of adjacent objects cannot be obtained between agents, $\mathrm{Wu}$ et al. [23] solved the problem of positive edge consistency in undirected networks, proposed an iterative linear LMI algorithm, and obtained the feedback matrix and the observer matrix. Through the output feedback protocol, Su et al. [24] studied the positive edge consistency of undirected and directed networks. Wang et al. [25] considered the input saturation problem of multiagent systems and adopted lowparameter, high-gain feedback techniques and high-gain observer methods to achieve consistent and robust control of multiagents. $\mathrm{Wu}$ and $\mathrm{Su}$ [26] studied the edge consistency problem of undirected network systems with positive constraints and input saturation. In contrast to the general conclusion of input saturation, the author improved the system consistency to a global level.

To sum up, the scholars performed substantial research on multimotor synchronous control $[27,28]$. However, the results in switching synchronous control are rare, especially for the study of motor load torque abrupt change which has always been the least of concern. Therefore, the current work explored the complex working conditions of the filling motor appearing in the filling multiservo motor system during the stop-start time when the load torque changes abruptly. It appropriately improves the filling motor model in accordance with actual production and proposes a relative coupling control strategy of multimotor with switching system structure. It then designs an equivalent SMC which can solve the poor coordination performance of multimachine in complex production conditions effectively during filling production and thus ensure the smooth operation of the system. The main research work of this paper can be summarized as following two points, consequently:

(1) This work proposes a multimotor system with a switching system structure which mainly addresses the complex working conditions of the filling motor during the stop-start time and the sudden change of the motor load torque. The system is optimized to make the model consistent with the actual engineering background.

(2) For the switching system structure of the filling motor, the common Lyapunov function is selected so that it could be switched at any initial state and reach the common sliding-mode surface, thereby ensuring the asymptotic stability of the multimotor system.

This paper is organized as follows: according to the structural advantages of the switching system, an improved relative coupling control strategy based on the filling multimotor system is designed in Section 2. The equivalent sliding-mode controller is designed to ensure single-axis error convergence and the multimotor system Lyapunov function is selected to ensure the progressive stability of the system in Section 3. Comparative simulations and analysis are illustrated in Section 4. Finally, this paper's conclusion is given in Section 5.

\section{Multimotor Cooperative Control Structure Based on Improved Relative Coupling}

In this section, a sliding-mode control method with a switching system structure for the filling multimotor system is designed. In the traditional control method generally, the processing of the sudden load of the motor is usually conservative. The control structure proposed in this section can improve the filling motor model to overcome the impact of the sudden change in load torque at the stop-start time, thereby tracking and controlling motor speed synchronously. The main advantage of designing the switching system is to solve a special working condition that occurs in the actual filling process; that is, the load torque of the filling motor jumped at the moment when the filling multimotor system is stopped-started, resulting in a large synchronization error in the system. When the switching system is designed, the filling motor can achieve smooth switching under complex working conditions when the load torque changes suddenly. At the same time, because the multimotor system selects the common Lyapunov function, it can ensure that the system reaches the common sliding-mode surface. It 
is gradually stabilized, so as to effectively overcome the impact of sudden change of load torque on the synchronization performance. The designed relative coupling control diagram of the $n$-improved motors is shown in Figure 1:

Because of the different functions of each station in the filling operation, the multimotor system is composed of a filling motor and a conveying motor. In Figure 1, considering that the system contains $n$ motors and motor 1 was the filling motor, other motors were taken as the transfer motors. The model of motor 1 is designed to switch the system structure. During the filling operation, the filling motor is stationary and stopped. Upon completion of the filling operation, the filling system needs to transport the filled finished product to the next station. Hence, the load torque of motor 1 is abruptly changed at the moment of stop-start, that is, changing from no-load to the increased load torque of the filling material which causes the filling motor to switch from subsystem 1 to subsystem 2. However, for the transmission motor system, the load torque does not change abruptly. Thus, a standard motor model can be used.

For the filling production transmission system, this study takes the permanent magnet synchronous motor as the research object. In the ideal permanent magnet synchronous model [29], the vector control strategy of $i_{d}=0$ is adopted to obtain the state equation of the $j$ motor in the multimotor system [30]:

$$
\left\{\begin{array}{l}
\frac{\mathrm{d} \theta_{j}}{\mathrm{~d} t}=\omega_{j}, \\
\frac{\mathrm{d} \omega_{j}}{\mathrm{~d} t}=-\frac{R_{\Omega j}}{J_{j}} \omega_{j}+\frac{3}{2} \cdot \frac{n_{p j}^{2} \psi_{f j}}{J_{j}} i_{q j}-\frac{T_{L j} n_{p j}}{J_{j}},
\end{array}\right.
$$

where $\theta_{j}$ is the rotor position; $\omega_{j}$ is the rotor electrical angular velocity; $T_{L j}$ is the load torque; $R_{\Omega j}$ is the rotational resistance coefficient of motor; $J_{j}$ is the moment of inertia; $n_{\mathrm{pj}}$ is the number of pole pairs of motor; $\psi_{f j}$ is the rotor flux; and $i_{d j}$ and $i_{q j}$ are the $d$-axis and $q$-axis components of the stator current.

Let $\left(R_{\Omega j} / J_{j}\right)=a_{j},(3 / 2) \cdot\left(n_{p j}^{2} \psi_{f j} / J_{j}\right)=b_{j}, \quad\left(T_{L j} n_{p j} / J_{j}\right)=$ $p_{j}$, and formula (1) can be expressed as follows:

$$
\dot{\omega}_{j}=-a_{j} \omega_{j}+b_{j} u_{j}-p_{j}, \quad j=1, k, k=2, \ldots, n,
$$

where $u_{j}=i_{q j}$ is the controller to be designed.

A filling motor model is established. Motor 1 is used as the filling motor. Because its load torque $T_{\mathrm{L} 1}$ is abrupt, the state equation of motor 1 with switching system structure is designed, mainly including two subsystems under different states of stop and start. From formula (2), we can obtain the following:

$$
\left\{\begin{array}{l}
f_{1}\left(\omega_{11}, t\right)=\dot{\omega}_{11}=-a_{1} \omega_{11}+b_{1} u_{1}, \\
f_{2}\left(\omega_{12}, t\right)=\dot{\omega}_{12}=-a_{1} \omega_{12}+b_{1} u_{1}-p_{1},
\end{array}\right.
$$

where $f_{1}\left(\omega_{11}, t\right)$ and $\omega_{11}$ are the subsystems and speeds when motor 1 is stopped, and the filling motor is no-load at this time; $f_{2}\left(\omega_{12}, t\right)$ and $\omega_{12}$ are the subsystems and speeds when motor 1 is started. Define the state variables as $x_{11}=\omega_{11}, x_{12}=\omega_{12}$, and rewrite formula (3) as matrix form as follows:

$$
\left(\begin{array}{l}
f_{1}\left(x_{11}, t\right) \\
f_{2}\left(x_{12}, t\right)
\end{array}\right)=\left(\begin{array}{l}
\dot{x}_{11} \\
\dot{x}_{12}
\end{array}\right)=-a_{1}\left(\begin{array}{l}
x_{11} \\
x_{12}
\end{array}\right)+b_{1}\left(\begin{array}{l}
u_{1} \\
u_{1}
\end{array}\right)-p_{1}\left(\begin{array}{l}
0 \\
1
\end{array}\right) .
$$

A transfer motor model is built, and the motors in formula (2) excluding motor 1 are redefined as

$$
\dot{\omega}_{k}=-a_{k} \omega_{k}+b_{k} u_{k}-p_{k}, \quad k=2, \ldots, n,
$$

where $\omega_{k}$ is the speed of the transfer motor in the multimotor system.

For a system consisting of a filling motor and a transfer motor, the tracking error of each motors is defined as follows:

$$
e_{j}^{*}=\omega_{j}^{*}-\omega_{j}, \quad j=1, k, k=2, \ldots, n,
$$

where $\omega_{j}^{*}$ and $\omega_{j}$ are the given speed value and actual output speed of the $j$ th motor of the system, respectively.

Define the synchronization error between the motors as follows:

$$
\tau_{j i}=\omega_{j}-\omega_{i}, \quad j=1, k, k=2, \ldots, n . i=1,2, \ldots, n, i \neq j,
$$

where $\tau_{j i}$ indicates the synchronization error between the $j$ th motor and the $i$ th motor.

In the relative coupling control strategy, the speed compensator is often used for feedback adjustment for the synchronization error. To eliminate the influence of different motor rotational inertia on the synchronization performance, the speed compensator module used a fixed gain compensator. The synchronization error compensation signal is as follows [31]:

$$
\Delta \omega_{j}=\sum_{i=1}^{n} \lambda_{j i} \tau_{j i}, \quad j=1, k, k=2, \ldots, n . i=1,2, \ldots, n, i \neq j,
$$

where $\lambda_{j i}$ is the ratio of the rotational inertia between adjacent motors. Its specific expression is

$$
\lambda_{j i}=\frac{J_{j}}{J_{i}} .
$$

\section{Sliding-Mode Controller Design and Stability Analysis}

3.1. Sliding-Mode Controller Design. This section designs a controller for a multimotor system to ensure that each axis has a better tracking and synchronization control effect. The equivalent sliding-mode controller is designed as follows [32]:

$$
\begin{aligned}
u_{j}= & \frac{\left(1+\sum_{i=1}^{n} \lambda_{j i}\right) a_{j} \omega_{j}+\dot{\omega}_{j}^{*}+\sum_{i=1}^{n} \lambda_{j i} \dot{\omega}_{i}}{\left(1+\sum_{i=1}^{n} \lambda_{j i}\right) b_{j}} \\
& +\frac{K_{j}}{b_{j}} \operatorname{sgn}\left(s_{j}\right), \quad j=1, k, k=2, \ldots, n, i=1,2, \ldots, n, i \neq j,
\end{aligned}
$$




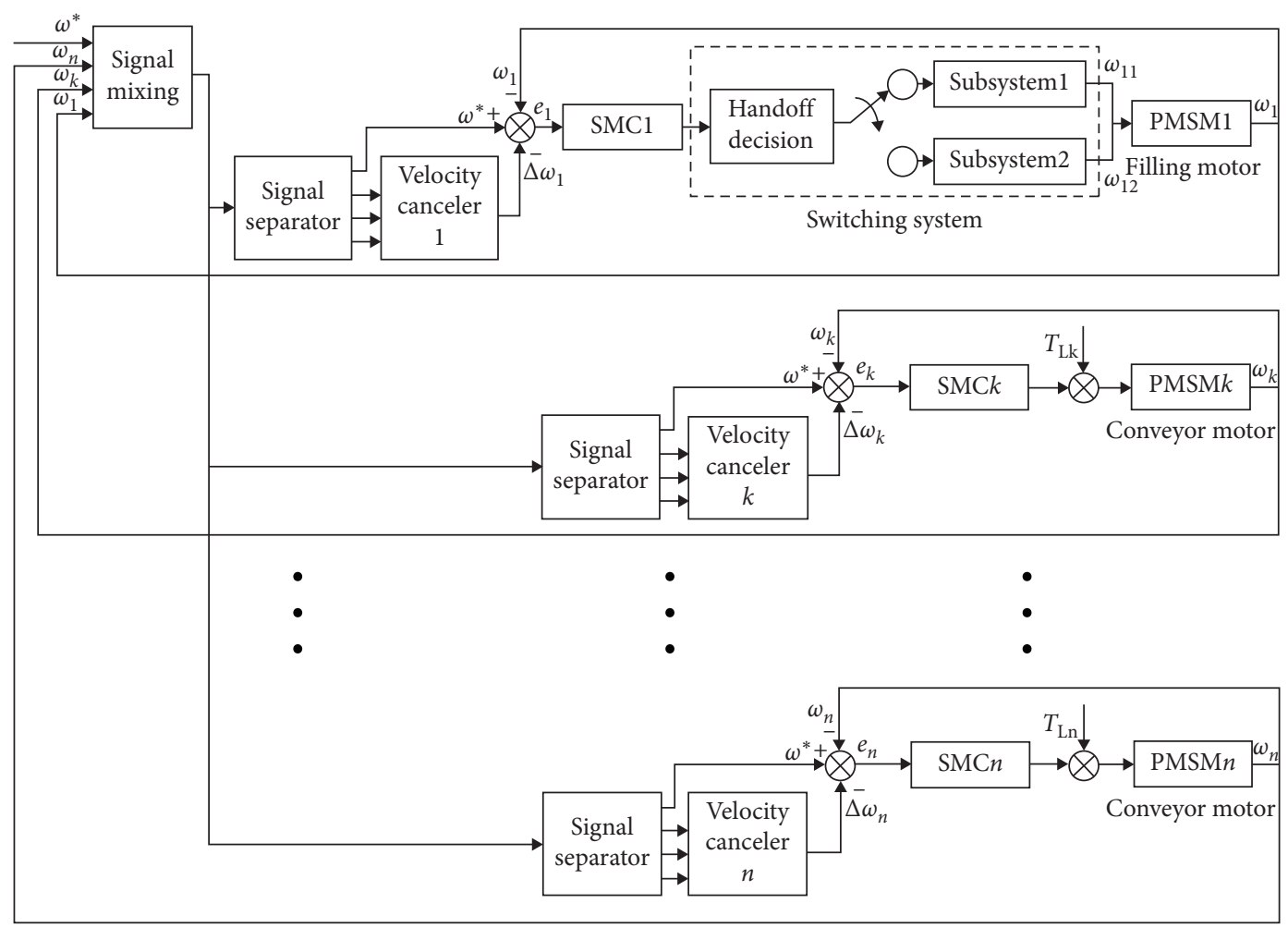

Figure 1: The designed relative coupling control diagram of the $n$-improved motors.

where $K_{j}$ is the sliding-mode coefficient to be designed and $s_{j}$ is the sliding surface to be designed.

Define the single-axis error of the multimotor synchronous system, and from formulas (6) and (8), we can obtain the following [17]:

$$
e_{j}=e_{j}^{*}-\Delta \omega_{j}=\omega_{j}^{*}-\omega_{j}-\Delta \omega_{j}, \quad j=1, k, k=2, \ldots, n .
$$

After the derivation of formula (11), we get

$$
\begin{aligned}
\dot{e}_{j} & =\dot{\omega}_{j}^{*}-\dot{\omega}_{j}-\sum_{i=1}^{n} \lambda_{j i}\left(\dot{\omega}_{j}-\dot{\omega}_{i}\right) \\
& =-\left(1+\sum_{i=1}^{n} \lambda_{j i}\right) \dot{\omega}_{j}+\dot{\omega}_{j}^{*}+\sum_{i=1}^{n} \lambda_{j i} \dot{\omega}_{i} .
\end{aligned}
$$

Define the sliding surface as follows:

$$
s_{j}=c_{j} e_{j}, \quad j=1, k \cdot k=2, \ldots, n .
$$

Design the sliding-mode parameter $c_{j}>0$, and calculate the derivation of formula (13) as follows:

$$
\dot{s}_{j}=c_{j} \dot{e}_{j}=c_{j}\left[-\left(1+\sum_{i=1}^{n} \lambda_{j i}\right) \dot{\omega}_{j}+\dot{\omega}_{j}^{*}+\sum_{i=1}^{n} \lambda_{j i} \dot{\omega}_{i}\right] .
$$

Let $\dot{s}_{j}=0$, and the equivalent control term $u_{\text {jeq }}$ is as follows:

$$
u_{\text {jeq }}=\frac{\left(1+\sum_{i=1}^{n} \lambda_{j i}\right) a_{j} \omega_{j}+\dot{\omega}_{j}^{*}+\sum_{i=1}^{n} \lambda_{j i} \dot{\omega}_{i}}{\left(1+\sum_{i=1}^{n} \lambda_{j i}\right) b_{j}} .
$$

Define the switching control term as follows:

$$
u_{\text {jsw }}=\frac{1}{b_{j}} K_{j} \operatorname{sgn}\left(s_{j}\right) \text {. }
$$

Thus, the sliding-mode control law of formula (10) consists of equivalent and switching control terms as follows:

$$
u_{j}=u_{\text {jeq }}+u_{\text {jsw }}
$$

3.2. Stability Analysis. The multimotor system Lyapunov function is selected, and this function can also be used as the public Lyapunov function of the switching system. Finally, results prove that the switching system can go from any initial state to the public sliding surface, thus ensuring the asymptotic stability of the entire system.

The stability of the transfer motor system is analyzed, and the Lyapunov function is selected:

$$
V_{k}=\frac{1}{2} s_{k}^{2}, \quad k=2, \ldots, n .
$$


After the derivation of formula (18), we get

$$
\begin{aligned}
\dot{V}_{k}= & s_{k} \dot{s}_{k}=s_{k} \cdot c_{k}\left[-\left(1+\sum_{i=1}^{n} \lambda_{k i}\right)\left(-a_{k} \omega_{k}+b_{k} u_{k}-p_{k}\right)\right. \\
& \left.+\dot{\omega}_{k}^{*}+\sum_{i=1}^{n} \lambda_{k i} \dot{\omega}_{i}\right] .
\end{aligned}
$$

Substituting formula (10) into (19) and reducing it results in

$$
\begin{array}{r}
\dot{V}_{k}=-s_{k} L_{k}\left(K_{k} \operatorname{sgn}\left(s_{k}\right)-p_{k}\right) \\
=-L_{k} K_{k}\left|s_{k}\right|+L_{k} p_{k} s_{k} \\
\leq-L_{k}\left(K_{k}-p_{k}\right)\left|s_{k}\right|,
\end{array}
$$

where

$$
L_{k}=c_{k}\left(1+\sum_{i=1}^{n} \lambda_{k i}\right)>0 .
$$

Design a proper sliding-mode coefficient $K_{k}>p_{k}+\eta_{k}$. $\eta_{j}$ is an arbitrarily small normal number which obtains

$$
\dot{V}_{k}<-L_{k} \eta_{k}\left|s_{k}\right|
$$

The sliding-mode principle [33] shows that the occurrence of the sliding-mode motion will cause $s_{k} \longrightarrow 0$ in limited time. According to formula (13), the single-axis error of each transfer motor $e_{k} \longrightarrow 0$ can be acquired.

The stability of the filling motor system is analyzed to select the common sliding-mode surface $s_{1}$ and the common Lyapunov function $V_{1}$ of the switching system:

$$
V_{1}=\frac{1}{2} s_{1}^{2}
$$

For the switching subsystem $f_{1}\left(x_{11}, t\right)$, derive formula (23) which gives

$$
\begin{aligned}
\dot{V}_{1} & =s_{1} \dot{s}_{1} \\
& =s_{1} \cdot c_{1}\left[-\left(1+\sum_{i=2}^{n} \lambda_{1 i}\right)\left(-a_{1} \omega_{11}+b_{1} u_{1}\right)+\dot{\omega}_{1}^{*}+\sum_{i=2}^{n} \lambda_{1 i} \dot{\omega}_{i}\right] .
\end{aligned}
$$

Substituting formula (10) into (24) and reducing it results in

$$
\dot{V}_{1}=-s_{1} L_{1} K_{1} \operatorname{sgn}\left(s_{1}\right)=-L_{1} K_{1}\left|s_{1}\right|
$$

where

$$
L_{1}=c_{1}\left(1+\sum_{i=2}^{n} \lambda_{1 i}\right)>0 .
$$

Design a proper sliding-mode coefficient $K_{1}>\eta_{1} ; \eta_{1}$ is an arbitrarily small normal number which obtains the following:

$$
\dot{V}_{1}<-L_{1} \eta_{1}\left|s_{1}\right|
$$

That is, the uniaxial error $e_{1}$ of the switching subsystem $f_{1}\left(x_{11}, t\right)$ gradually converges to 0 .

For the switching subsystem $f_{2}\left(x_{12}, t\right)$, just design the appropriate sliding-mode coefficient $K_{1}>p_{1}+\eta_{1}$ that can obtain formula (27) in the same way; that is, the uniaxial error $e_{1}$ of the switching subsystem $f_{2}\left(x_{12}, t\right)$ gradually converges to 0 . The common Lyapunov function shows that $\dot{V}_{1}$ exists regardless of the subsystem activated. Thus, the sliding-mode function $s_{1} \neq 0$ is attenuated. To sum up, when $t \longrightarrow \infty, e_{j} \longrightarrow 0, j=1, k k=2, \ldots, n$.

The convergence of tracking error and synchronization error is to be proved as follows [34].

From simultaneous formulas (6), (8), and (11), the following can be obtained:

$$
e_{j}=\left(1+\sum_{i=1}^{n} \lambda_{j i}\right) e_{j}^{*}-\sum_{i=1}^{n} \lambda_{j i} e_{i}^{*} .
$$

Rewrite formula (28) as matrix form:

$$
\left(\begin{array}{c}
e_{1} \\
\vdots \\
e_{j} \\
\vdots \\
e_{n}
\end{array}\right)=\left(\begin{array}{ccccc}
1+\sum_{i=1}^{n} \lambda_{1 i} & \cdots & -\lambda_{1 i} & \cdots & -\lambda_{1 n} \\
\vdots & \ddots & & & \vdots \\
-\lambda_{j 1} & & 1+\sum_{i=1}^{n} \lambda_{j i} & & -\lambda_{j n} \\
\vdots & & & \ddots & \vdots \\
-\lambda_{n 1} & \cdots & -\lambda_{n i} & \cdots & 1+\sum_{i=1}^{n} \lambda_{n i}
\end{array}\right)\left(\begin{array}{c}
e_{1}^{*} \\
\vdots \\
e_{j}^{*} \\
\vdots \\
e_{n}^{*}
\end{array}\right) .
$$

Define the matrix of coefficient $G_{n \times n}$ as

$$
G_{n \times n}=\left(\begin{array}{ccccc}
1+\sum_{i=1}^{n} \lambda_{1 i} & \cdots & -\lambda_{1 i} & \cdots & -\lambda_{1 n} \\
\vdots & \ddots & & & \vdots \\
-\lambda_{j 1} & & 1+\sum_{i=1}^{n} \lambda_{j i} & & -\lambda_{j n} \\
\vdots & & & \ddots & \vdots \\
-\lambda_{n 1} & \cdots & -\lambda_{n i} & \cdots & 1+\sum_{i=1}^{n} \lambda_{n i}
\end{array}\right) .
$$

The coefficient matrix $G_{n \times n}$ is a nonsingular matrix. Because $e_{j} \longrightarrow 0$ has been proven above, $\left[\begin{array}{lllll}e_{1}^{*} & \cdots & e_{j}^{*} & \cdots & e_{n}^{*}\end{array}\right]^{T}$ can be obtained with only unique zero solution. Therefore, the tracking error of each axis can be converged to 0 , and the synchronous compensation signal $\Delta \omega_{j}$ also converges to 0 .

The proof is completed.

\section{Simulation and Experimental Results Analysis}

To verify the theoretical research and control method in this paper, this section uses Matlab/Simulink to perform a 
TABLE 1: The physical parameter setting of each motor.

\begin{tabular}{lccc}
\hline Parameter & Motor 1 & Motor 2 & Motor 3 \\
\hline$R_{\Omega}(\mathrm{N} \cdot \mathrm{m} \cdot \mathrm{s})$ & $4.831 \times 10^{-5}$ & $4.846 \times 10^{-5}$ & $4.827 \times 10^{-5}$ \\
$\psi_{f}(\mathrm{~Wb})$ & 0.175 & 0.173 & 0.178 \\
$n_{\mathrm{p}}$ & 2 & 2 & 2 \\
$J\left(\mathrm{~kg} \cdot \mathrm{m}^{2}\right)$ & $8.5 \times 10^{-4}$ & $8.47 \times 10^{-4}$ & $8.51 \times 10^{-4}$ \\
\hline
\end{tabular}

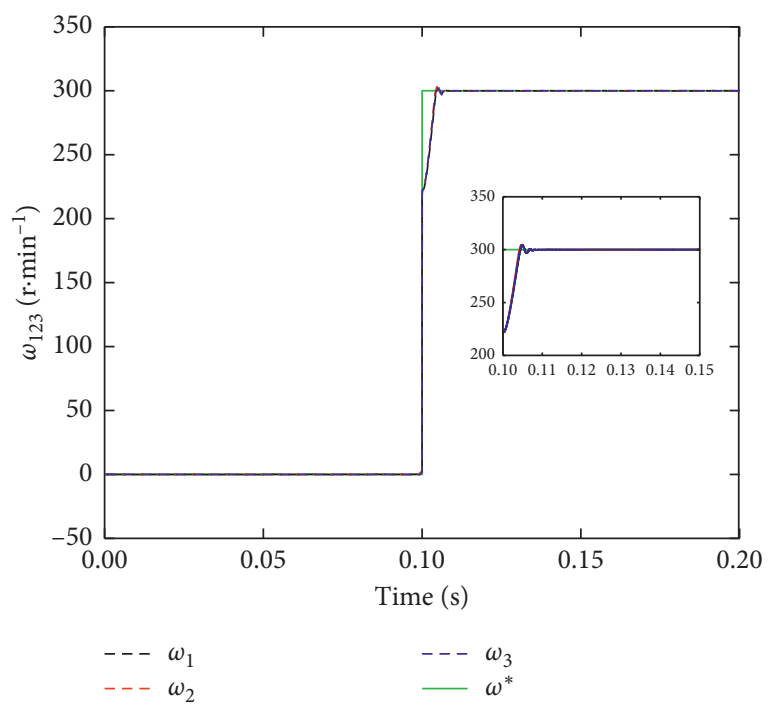

(a)

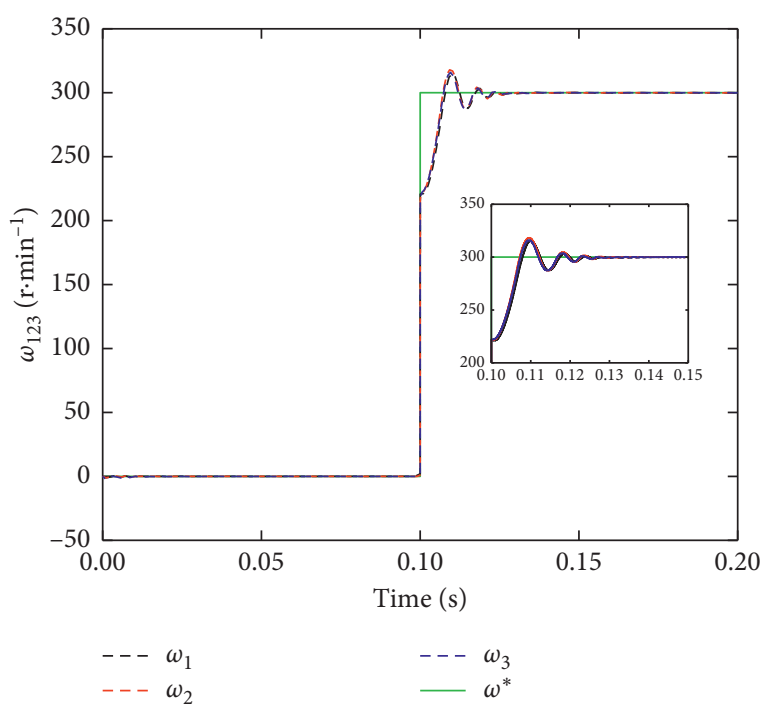

(b)

Figure 2: Comparative simulation results of the speed follow of each motor.

simulation analysis of the system and thus verify the research of multimotor cooperative control under complex working conditions of filling production. At the same time, to highlight the advantages of the improved control structure, this section also adds a comparative experiment simulation. Under the traditional control structure, the PI controller is used to adjust the system error, and the sudden load torque is also applied to the filling motor. For the stop-start process during the filling system operation, the speed given curve of the designed multimotor system is as follows:

$$
\omega^{*}= \begin{cases}0, & 0 \leq t \leq 0.1, \\ 300, & 0.1<t \leq 0.2\end{cases}
$$

The physical parameter setting of each motor used in Matlab/Simulink is shown in Table 1:

The entire experimental simulation duration is set to $0.2 \mathrm{~s}$. For the experimental simulation of the improved control structure, designed motor 1 is switched in $0.1 \mathrm{~s}$, and the load torque of the given subsystem $f_{2}\left(x_{12}, t\right)$ is $1 \mathrm{~N} \cdot \mathrm{m}$. For the experimental simulation of the traditional control structure, a load torque $1 \mathrm{~N} \cdot \mathrm{m}$ is suddenly applied to motor 1 at $0.1 \mathrm{~s}$. To simulate the process of conveying the empty bottle to the filling station by the transfer motor, the load torque of given motor 2 is $0.1 \mathrm{~N} \cdot \mathrm{m}$. To simulate the process of conveying the finished product to the next station by transfer the motor, the load torque of the given motor 3 is
$1 \mathrm{~N} \cdot \mathrm{m}$. In the controller, the sliding-mode coefficient is $K_{1}=K_{2}=K_{3}=10000$, and the sliding surface parameter is $c_{1}=c_{2}=c_{3}=1000$. Figure 2 is the comparison simulation diagrams of the speed following curves of each motors.

Figure 2 is the speed following curve diagrams of each motor. Among them, (a) and (b) show the running trajectory of each motor speed under the improved and traditional relative coupling control strategy, respectively. The designed system speed-given curve simulates the stop-start process of filling production, and $0.1 \mathrm{~s}$ is the speed jump time. Analysis and simulation results show that, in comparison with the traditional control structure, the speed of motor 1 with the switching system structure runs more stably at the initial stage and the switching time. In addition, the rising curve is smoother, and the speeds of motors 2 and 3 can also be better tracked with the fixed value of the load torque, especially because the controller adjustment time only needs about $0.01 \mathrm{~s}$ at the time of switching, with no obvious overshoot of the speed.

(a), (b), and (c) in Figure 3 are the simulation comparison diagrams of the rotational speed-tracking errors of motors 1, 2, and 3, respectively. Among them, the red dashed line and the blue solid line, respectively, represent the tracking error under the traditional and the improved control strategy, and a supplementary explanation is made to Figure 2. Analysis and simulation results show that, Figure 3 


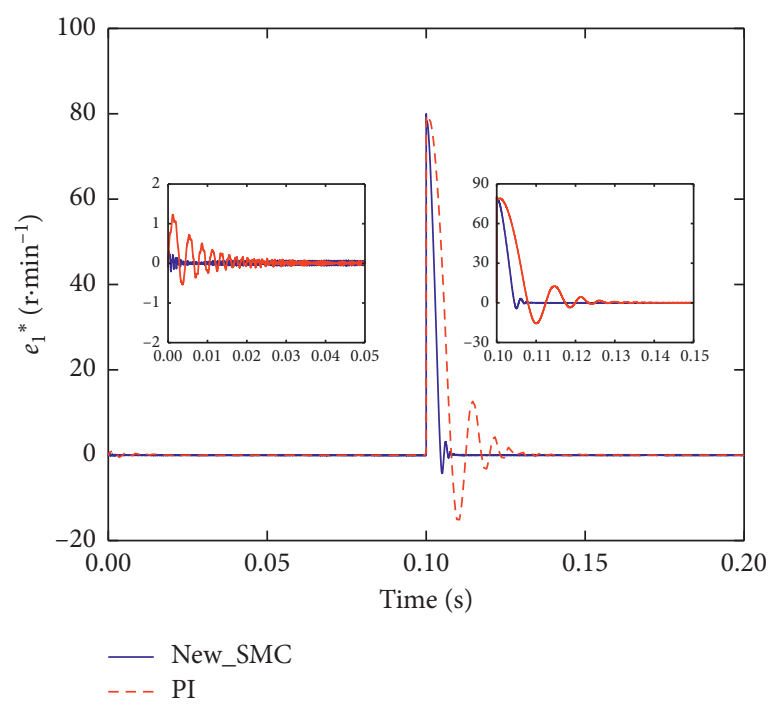

(a)

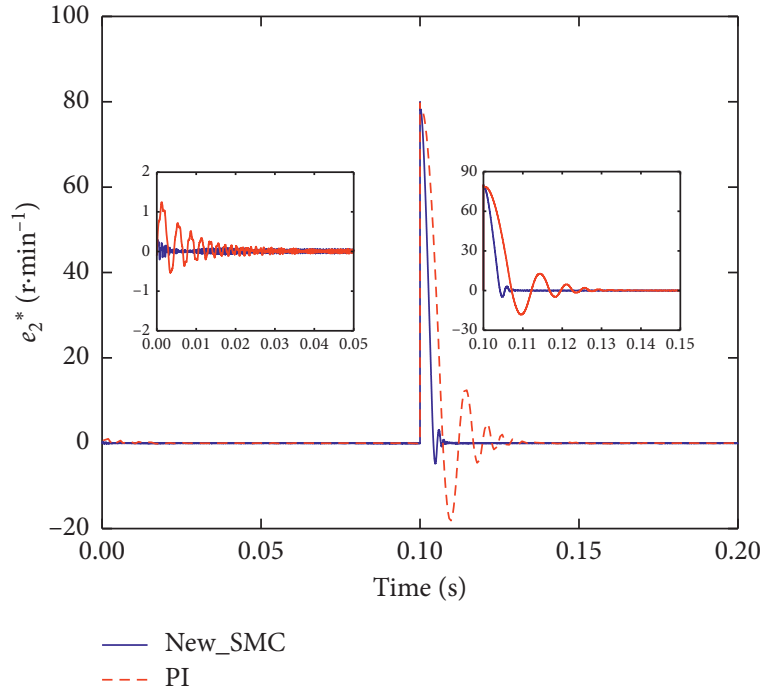

(b)

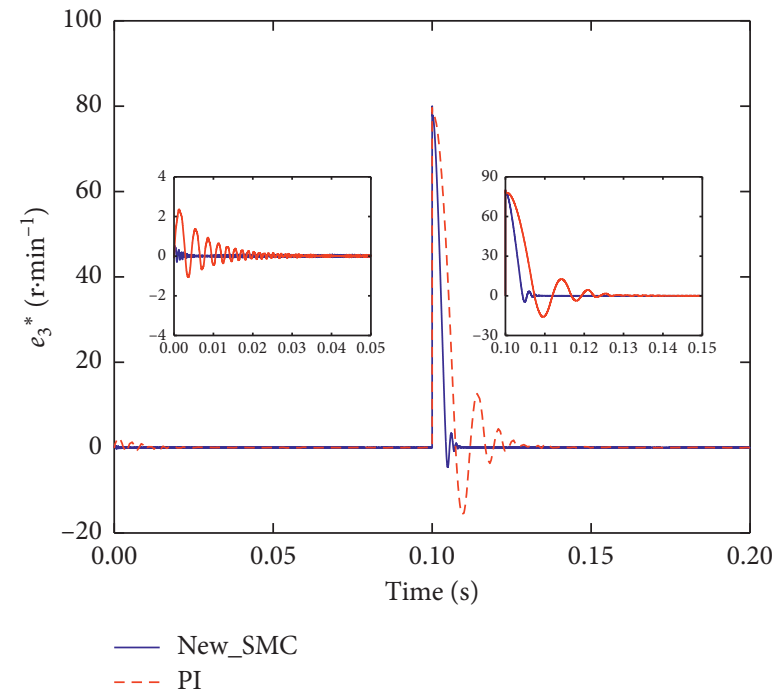

(c)

FIgURE 3: Comparative simulation results of tracking errors of each motor.

shows the situation of each motor tracking a given value more intuitively. At the same time, each motor in the improved control structure can achieve rapid convergence of the tracking error within $0.01 \mathrm{~s}$, especially at the stop-start time of $0.1 \mathrm{~s}$, when the impact of load torque mutation has good robustness. However, in the initial stage within $0 \sim 0.1 \mathrm{~s}$, the tracking error jitter of motor 3 is larger than that of other motors, which is mainly caused by the larger load torque than that of other motors. At the moment of stop-start, the set value of the speed of each motor suddenly jumps from $0 \mathrm{rad} \cdot \mathrm{min}^{-1}$ to $300 \mathrm{rad} \cdot \mathrm{min}^{-1}$, and the actual motor speed value cannot jump, so a large tracking error (about $80 \mathrm{rad} \cdot \mathrm{min}^{-1}$ ) will occur in $0.1 \mathrm{~s}$. On the other hand, it also confirmed the authenticity and rationality of the simulation.

(a), (b), and (c) in Figure 4 are the simulation comparison diagrams of the synchronous errors between motors
1 and 2, between motors 1 and 3 and between motors 2 and 3 , respectively. Among them, the red dashed line and the blue solid line represent the synchronous error under the traditional and the improved control strategy, respectively. In terms of error suppression time, the synchronous error among the motors in the improved control structure can be quickly converged within $0.01 \mathrm{~s}$. However, in the traditional control structure, the error will not converge until about $0.15 \mathrm{~s}$. In terms of error suppression effect, the improved method proposed in this paper can make the error jitter suppression is more significant and change amplitude is smaller. Among them, motors 1 and 2 have a smaller initial synchronous error in $0 \sim 0.1 \mathrm{~s}$, and it also includes the case where the error curve between the motors is significantly different at the switching time of $0.1 \mathrm{~s}$. This result is mainly caused by the large difference in load torque of each motor, and it is real and reasonable. 


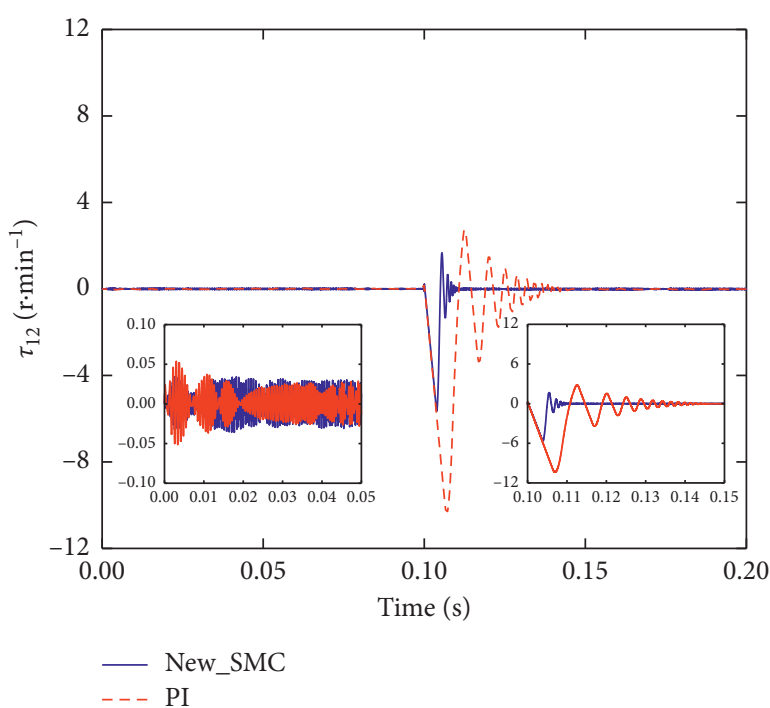

(a)

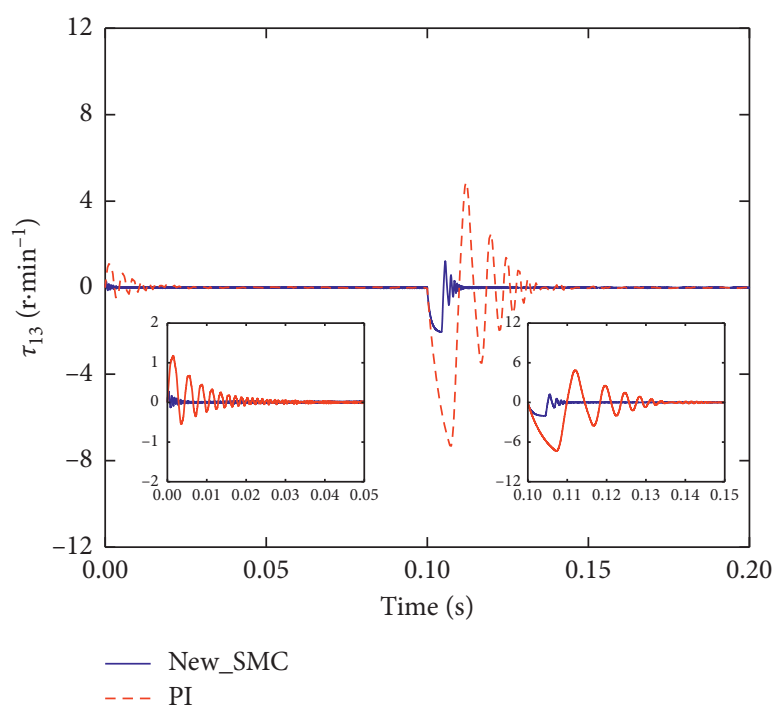

(b)

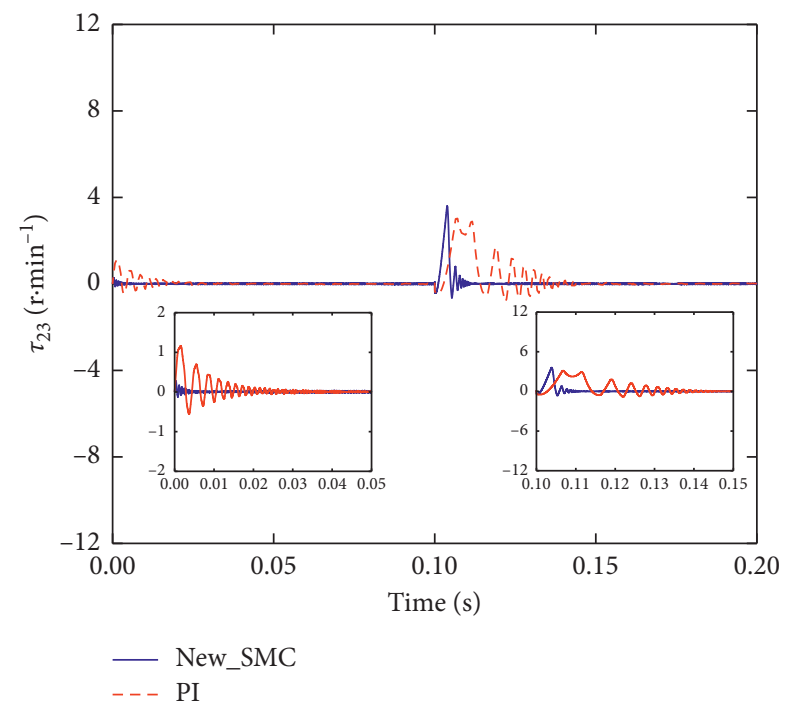

(c)

FIGURE 4: Comparative simulation results of synchronization errors of each motor.

\section{Conclusions}

To realize the follow-up control and synchronous control of the filling multimotor system under complex working conditions, the relative coupling control strategy including the switching system structure is firstly established. Secondly, the common sliding-mode surface is selected based on the filling motor, and the equivalent sliding-mode controller is designed. Thirdly, by selecting the common Lyapunov function, the switching system can reach the common sliding-mode surface in any initial state. Hence, the entire system reaches an asymptotic stable state. By adopting the proposed method, the adverse effects of sudden changes in load torque can be effectively weakened. As such, the system has better dynamic and static characteristics and is particularly robust to torque parameter jumps that occur during the switching process. Simulation results verify the effectiveness of this method for multimotor collaborative control of filling.

\section{Data Availability}

The model data used to support the findings of this study are available from the corresponding author upon request.

\section{Conflicts of Interest}

The authors declare that there are no conflicts of interest regarding the publication of this paper.

\section{Acknowledgments}

The study was supported by the National Key R\&D Program of China (no. 2018YFD0400705). 


\section{References}

[1] H. Yan, Y. Tian, H. Li, H. Zhang, and Z. Li, "Input-output finite-time mean square stabilisation of nonlinear semiMarkovian jump systems," Automatica, vol. 104, pp. 82-89, 2019.

[2] H. Su, Y. Ye, Y. Qiu, Y. Cao, and M. Z. Q. Chen, "Semi-global output consensus for discrete-time switching networked systems subject to input saturation and external disturbances," IEEE Transactions on Cybernetics, vol. 49, no. 11, pp. 3934-3945, 2019.

[3] H. Su, J. Zhang, and X. Chen, "A stochastic sampling mechanism for time-varying formation of multi-agent systems with multiple leaders and communication delays," IEEE Transactions on Neural Networks and Learning Systems, vol. 30, no. 12, pp. 3699-3707, 2019.

[4] S. Mao, H. Wu, M. Lu, and C.-W. Cheng, "Multiple 3D marker localization and tracking system in image-guided radiotherapy," International Journal of Robotics and Automation, vol. 32, no. 5, pp. 517-523, 2017.

[5] C. Xu, Y. Zhao, B. Qin, and H. Zhang, "Adaptive synchronization of coupled harmonic oscillators under switching topology," Journal of the Franklin Institute, vol. 356, no. 2, pp. 1067-1087, 2019.

[6] C.-F. Zhang, Y.-Y. Xiao, J. He, and M. Yan, "Improvement of electronic line-shafting control in multi-axis systems," International Journal of Automation and Computing, vol. 15, no. 4, pp. 474-481, 2018.

[7] H. Yang, Y. Fu, and D. Wang, "Multi-ANFIS model based synchronous tracking control of high-speed electric multiple unit," IEEE Transactions on Fuzzy Systems, vol. 26, no. 3, pp. 1472-1484, 2018.

[8] W. Zhao and H. Zhang, "Coupling control strategy of force and displacement for electric differential power steering system of electric vehicle with motorized wheels," IEEE Transactions on Vehicular Technology, vol. 67, no. 9, pp. 8118-8128, 2018.

[9] T. Shi, H. Liu, Q. Geng, and C. Xia, "An improved relative coupling control structure for multi-motor speed synchronous driving system," IET Electric Power Applications, vol. 10, no. 6, pp. 451-457, 2016.

[10] C. Sun, G. Gong, H. Yang, and F. Wang, "Fuzzy sliding mode control for synchronization of multiple induction motors drive," Transactions of the Institute of Measurement and Control, vol. 41, no. 11, pp. 3223-3234, 2019.

[11] C. Zhang, H. Wu, B. Liu, J. He, and B. Li, "Hybrid structure based tracking and consensus for multiple motors," Mathematical Problems in Engineering, vol. 2014, Article ID 967578, 9 pages, 2014

[12] L. Li, L. Sun, and S. Zhang, "Mean deviation coupling synchronous control for multiple motors via second-order adaptive sliding mode control," ISA Transactions, vol. 62, pp. 222-235, 2016.

[13] K. Huang, Y. Zhang, Y. Li, C. Yang, and Z. Wang, "Effects of extenal forcing on evolutionary games in complex networks," Choas: An Interdisciplinary Journal of Nonlinear Science, vol. 28, no. 9, Article ID 093108, 2018.

[14] J. He, X. Chen, S. Mao, C. Zhang, and J. Liu, "Virtual line shafting-based total-amount coordinated control of multimotor traction power," Journal of Advanced Transportation, vol. 2020, Article ID 4735397, 9 pages, 2020.

[15] L.-B. Li, L.-L. Sun, S.-Z. Zhang, and Q.-Q. Yang, "Speed tracking and synchronization of multiple motors using ring coupling control and adaptive sliding mode control," ISA Transactions, vol. 58, pp. 635-649, 2015.

[16] C. Xia, Y. Yan, B. Ji, and T. Shi, "Two-degree-of-freedom proportional integral speed control of electrical drives with Kalman-filter-based speed estimation," IET Electric Power Applications, vol. 10, no. 1, pp. 18-24, 2016.

[17] J. He, X. Jiang, C. Zhang et al., "Multiaxis servo synergic control based on sliding mode controller," Journal of Control Science and Engineering, vol. 2019, Article ID 9249270, 16 pages, 2019.

[18] D. Yang, W. Ren, X. Liu, and W. Chen, "Decentralized eventtriggered consensus for linear multi-agent systems under general directed graphs," Automatica, vol. 69, pp. 242-249, 2016.

[19] C. Zhang, H. Wu, J. He, and C. Xu, “Consensus tracking for multi-motor system via observer based variable structure approach," Journal of the Franklin Institute, vol. 352, no. 8, pp. 3366-3377, 2015.

[20] C. Xia, L. Li, G. Xin, and T. Shi, "Speed synchronization control of dual-PMSM system," Diangong Jishu Xuebao/ Transactions of China Electrotechnical Society, vol. 32, no. 23, pp. 1-8, 2017.

[21] M. Wang, X. Ren, and Q. Chen, "Robust tracking and distributed synchronization control of a multi-motor servomechanism with $\mathrm{H}$-infinity performance," ISA Transactions, vol. 72, pp. 147-160, 2018.

[22] L. Liu, D. Wei, and B. Zhang, "Synchronization control of chaos in complex motor networks with small-world topology based on dynamic relaying," Jisuan Wuli/Chinese Journal of Computational Physics, vol. 35, no. 6, pp. 750-756, 2018.

[23] H. Wu, J. Lam, and H. Su, "Global consensus of positive edge system with sector input nonlinearities," IEEE Transactions on Systems, Man, and Cybernetics: Systems, pp. 1-10, 2019.

[24] H. Su, H. Wu, and J. Lam, "Positive edge-consensus for nodal networks via output feedback," IEEE Transactions on Automatic Control, vol. 64, no. 3, pp. 1244-1249, 2018.

[25] X. Wang, H. Su, M. Z. Q. Chen, and X. Wang, "Observerbased robust coordinated control of multiagent systems with input saturation," IEEE Transactions on Neural Networks and Learning Systems, vol. 29, no. 5, pp. 1933-1946, 2018.

[26] H. Wu and H. Su, "Positive edge consensus of networked systems with input saturation," ISA Transactions, vol. 96, pp. 210-217, 2020.

[27] J. He, B. Yang, C. Zhang et al., "Robust consensus braking algorithm for distributed EMUs with uncertainties," IET Control Theory \& Applications, vol. 13, no. 17, pp. 2766-2774, 2019.

[28] C. Xu, H. Su, C. Liu, and G. Zhang, "Robust adaptive synchronization of complex network with bounded disturbances," Advances in Difference Equations, vol. 2019, no. 1, 2019.

[29] P. Gao, G. Zhang, H. Ouyang, and L. Mei, “A sliding mode control with nonlinear fractional order PID sliding surface for the speed operation of surface-mounted PMSM drives based on an extended state observer," Mathematical Problems in Engineering, vol. 2019, Article ID 7130232, 13 pages, 2019.

[30] J. He, J. Ding, C. Zhang et al., "Research on multi-axis servo synergic control system based on sliding mode variable structure," in Proceedings of the IECON 2019-45th Annual Conference of the IEEE Industrial Electronics Society, pp. 6908-6915, Lisbon, Portugal, October 2019.

[31] Y. Xia, K. Xu, Y. Li, G. Xu, and X. Xiang, "Modeling and threelayer adaptive diving control of a cable-driven underwater parallel platform," IEEE Access, vol. 6, pp. 24016-24034, 2018. 
[32] Y. Pan, M. J. Er, T. Sun, B. Xu, and H. Yu, “Adaptive fuzzy PD control with stable $H^{\infty}$ tracking guarantee," Neurocomputing, vol. 237, pp. 71-78, 2016.

[33] V. I. Utkin, Sliding Modes in Control and Optimization, Springer-Verlag, Berlin, Germany, 1992.

[34] J. He, L. Mi, J. Liu, X. Cheng, Z. Lin, and C. Zhang, "Ring coupling-based collaborative fault-tolerant control for multirobot actuator fault," International Journal of Robotics and Automation, vol. 33, no. 6, pp. 672-680, 2018. 